\title{
A Research on Interior Location of Improved Monte Carlo Algorithm Based on RSSI
}

\author{
LiWen Liang ${ }^{1}$ \\ Beijing University of Civil Engineering and Architecture \\ Beijing, 100044,China \\ E-mail: IIw_work@163.com
}

\section{Zhi Tan}

Beijing University of Civil Engineering and Architecture

Beijing, 100044, China

E-mail: tanzhi@bucea.edu.cn

For the problem that the traditional static positioning algorithms can not locate the mobile network node accurately, and the traditional Monte Carlo localization algorithm features low positioning accuracy and poor positioning accuracy due to the low sampling efficiency of the nodes, an improved Monte Carlo (Higher Monte Carlo, HMCL) localization algorithm is thereby proposed. The RSSI distance measured model is used to optimize the sampling weight and the position of the node is predicted by the least squares fitting nodes. At the same time, the cross algorithm is introduced to improve the particle activity; finally, the sampling area is optimized and the sampling interval is determined. It is found that the modified Monte Carlo algorithm can greatly reduce the number of sampling times and the positioning error of the nodes; at meanwhile, the positioning error is stable.

CENet2017

22-23 July, 2017

Shanghai, China

\section{${ }^{1}$ Speaker}




\section{Introduction}

The general positioning technology can not meet the accuracy of its requirements in indoor due to the particularity of the indoor environment. The wireless sensor network, characterized by low power consumption and high organization, has become an important part of indoor positioning technology [1]. The wireless sensor network is located by a large number of randomly distributed unknown nodes and anchor nodes. The newly positioned nodes are used as new anchor nodes to locate other unknown nodes. This cooperation is conducive in terms of rapid positioning and good network stability. The authors proposed different algorithms such as MCL (Monte Carlo Localization) localization algorithm, centroid localization algorithm, DVHop algorithm, RSS (Received Signal Strength), TOA (Time Of Arrival), TDOA (Time Difference Of Arrival ), AOA (Angle Of Arrival) and other positioning algorithms.

MCL (Monte Carlo Localization) positioning algorithm is a classic mobile node location method, but this algorithm requires exessive sampling data. It is a brand new idea to predict the motion trajectory of the mobile node by using the least squares so as to optimize the traditional MCL localization algorithm [2].in order to improve the accuracy of prior estimation, the first few moments of trajectory prediction motion parameters of current for the moment were proposed[3]. The method of sample particle crossover was used to accelerate the algorithm of sampling effective particles, which can accelerate the convergence speed of the algorithm obviously, The history anchor nodes with RSSI nodes are positioned narrow location sampling range. Based on the current RSSI ranging neighbor distinguishing effective sampling point weights, this method has greatly improved the sampling range[4].

In this paper,an improved Monte Carlo (Higher Monte Carlo, HMCL) localization algorithm is proposed to optimize the sampling weights by the RSSI distance measurement model, and the location of nodes is predicted by the least squares fitting nodes. Then, the cross algorithm is introduced to increase particle mobility. Experiments show that modified Monte Carlo algorithm can greatly reduce the positioning error of the nodes.

\section{Logarithmic-normal Distribution Model}

The RSSI is sent by the beacon node and measured by an unknown node that receives the signal. The signal reception strength is equal to the signal emission intensity minus the signal propagation loss without regard to the signal gain. The free space model and the logarithmicnormal distribution model are the two most commonly used models of signal propagation loss [5]. In this paper, we use log-normal distribution model to study.

The logarithmic-constant distribution model is shown in Equation (2.1)

$$
\frac{\operatorname{Pr}(d)}{\operatorname{Pr}\left(d_{0}\right)}=10 * k * \log \left(\frac{d}{d_{0}}\right)+X_{\text {ᄎ }}
$$

$P_{r}\left(d_{0}\right)$ refers to the intensity loss when the signal propagation distance is $d_{0}$ in the ideal space; Gaussian random function with an average value of 0 , which reflects the intensity loss of the signal propagation distance $\mathrm{d}$, distance from a certain time, the signal strength loss range; $\mathrm{k}$ refers to the signal strength loss coefficient, the general value between 2-5.

\section{Monte Carlo localization algorithm}

The Monte Carlo localization algorithm is a particle filter process used to represent the probability model of perception and motion, which can be more accurately positioned in 
complex network positioning [6]. The positioning phase includes prediction and filtering. The prediction phase is based on the velocity information of the node and the sample location is determined by the previous information. The particle location information is obtained by random sampling. In the filtering phase, the particles obtained in the prediction phase are screened by the anchor node information, the particles that do not satisfy the condition are discarded, the particles that can meet the filter conditions are recorded, and their mean values are used to estimate the position of the nodes.

\section{Improved Positioning Monte Carlo Algorithm}

In general, it may be difficult to use the variation of the variable formula to calculate the distribution of the spatial function. A simple but powerful alternative is the Monte Carlo method. In this paper, an improved Monte Carlo (Higher Monte Carlo, HMCL) localization algorithm is proposed.

\subsection{Weight Optimization Based on RSSI}

The receiver signal strength device features low cost and easiness of implementation. RSSI weight position estimation is divided into two stages without weight position estimation and weighting position estimation. In the non-weight position estimation phase, the ordinary node carries out the average position estimation according to the effective sampling point that has been filtered and sends the position estimate and its error measure to the common neighbor node. In the weighted position estimation stage, the common node according to the neighbor anchor node information and Neighbor node location estimates and their errors are measured by RSSI distance calculation weights, updating the estimated location[7].

As shown in Figure 1, D is the unknown node, B and C are the anchor nodes, A is the D's neighbor node, the circle with $\mathrm{B}$ as the center of circle and the circle with $\mathrm{C}$ as the center of the overlap is the $\mathrm{E}$ point of the location of the sampling effective range, $\mathrm{E} ; \mathrm{F}$ is the effective range of two position samples. We can estimate the distance from the unknown nodes to the distance between the unknown nodes and the RSSI of the neighbor nodes [8]. The distance from the mark D to the A, B and C refers to $\mathrm{d} 1, \mathrm{~d} 2$ and $\mathrm{d} 3$ respectively, and the RSSI distance estimation error is $\partial$. Let the node error be $e_{1}$, shown as follows.

$$
\begin{aligned}
& \sqrt{\left(x_{D}-x_{A}\right)^{2}+\left(x_{D}-x_{A}\right)^{2}} \leqslant d_{1}+\partial+e_{1} \\
& \sqrt{\left(x_{D}-x_{B}\right)^{2}+\left(x_{D}-x_{B}\right)^{2}} \leqslant d_{2}+\partial \\
& \sqrt{\left(x_{D}-x_{C}\right)^{2}+\left(x_{D}-x_{C}\right)^{2}} \leqslant d_{3}+\partial
\end{aligned}
$$

Figure 1 : Distance between Nodes

If the node satisfies Formula (4.1), $w_{i 1}=1$; if not, $w_{i 1}=0$.If the number of anchor nodes in the communication range of the unknown node is less than 3 , the number of the neighbor nodes is the smallest, and then the neighbor node is used to calculate the weight of the neighbor node and the common node. If the total number of neighbor nodes and common nodes 
is less than three, the weight estimation is not performed and the position estimation of the unknown node is estimated directly at the position without weight in the first stage.

\subsection{Particle Production based on Sample Crossover}

After observation of the particles and the weighted calculation, the weight of many particles are very close to 0 , demanding re-position update and weight calculation after several iterations have achieveda certain number of weights close to 1 particle. In order to accelerate the acquisition of particles with a weight close to 1, the algorithm randomly selects two particles for cross-operation from particles satisfying the filter condition, ie, the weight is close to 1 . In general, the linear cross method is used here[9], such as Formula (4.2).

$$
x_{p}=a x_{i}+(1-a) x_{j} \quad y_{p}=a y_{i}+(1-a) y_{j}
$$

\subsection{Prediction of Motion Trends by Least Square Method}

Using the least squares fitting method to predict the trend of node movement, when the new location information is generated, the first three moments of the earliest position information are replaced; in order to constantly update the node's latest three historical locations, assuming that the first three moments of the node be , through three time positions, fitting node. At the position of the vertical and horizontal coordinates, the horizontal and vertical coordinates are solved, and finally the particle size and direction are predicted. The predicted psotion of node is obtained [10]. Formula(4.3) is obtained.

$$
w_{i}=1-\frac{n * 5}{a+n * 5} *\left(1+w_{i l}\right)
$$

Finally, the position of the unknown node at time $t$ is shown in Equation (4.4)

$$
x_{t}=x_{i} * x_{p}, \quad y_{t}=y_{i} * y_{p}
$$

\subsection{Prediction of Motion Trends by Least Square Method}

The system work flow chart is shown below.

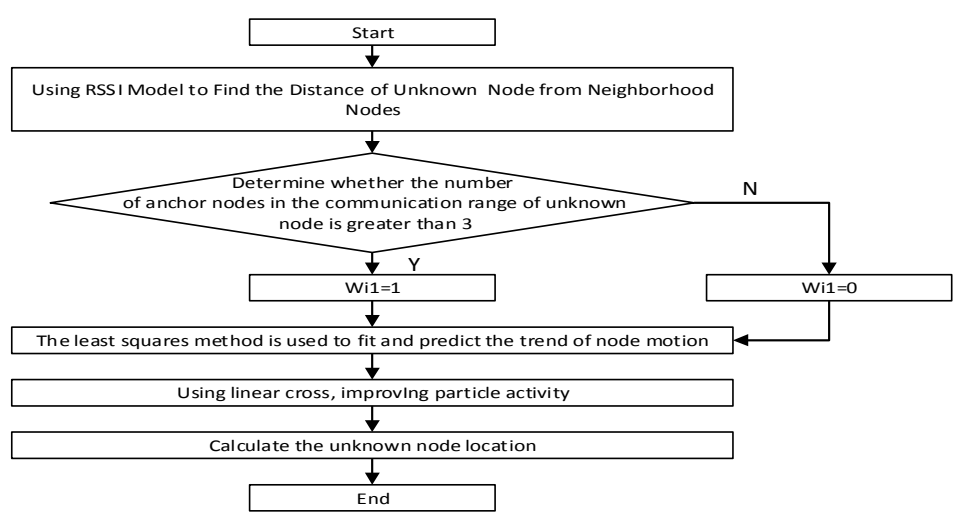

Figure 2 : System Work Flow Chart

\section{Experimental Verification and Simulation}

In order to verify the algorithm, the CC2430 experimental platform is used to record the RSSI value, and the distance between the anchor nodes and the unknown nodes is calculated by the model. 312 rooms are involved in the experiment. However, as the experimental equipment and the experimental site are effective, in order to verify the algorithm on a large scale, the software simulation is carried out to verify the consistency of the two. 


\subsection{Experimental Platform Simulation}

The platform uses the node CC2430 to measure the RSSI value by using the log-Changshu model. The relationship between RSSI and distance is determined by Equation (2.1), The attenuation factor $\mathrm{k}$ is 2 and the reference distance do $=1 \mathrm{~m} . \quad X_{\lambda}$ is a random number that follows the Gaussian distribution, and the standard deviation is 11.8 . The experiment area is $7 \mathrm{~m}$ $\times 3.5 \mathrm{~m}$. With 3 unknown nodes and 4 anchor nodes to locate where the error expression is $e_{3}$ at the moving speed of $0.5 \mathrm{~m} / \mathrm{s}$, the test were carried out by 5 times, 10 times, 15 times, 20 times, etc. unitl 50 times. The system error comparison chart shown in Table1, where the average error expression is represented as follows, $\mathrm{s}$ is the number of tests, $\left(x_{t}, y_{t}\right)$ is the calculated position of node and $(x, y)$ is the actual position of node. The results are shown in Table 1 .

$$
e_{2}=\sqrt{\left(x-x_{t}\right)^{2}+\left(y-y_{t}\right)^{2}} \quad e_{3}=\sum e_{2} / s
$$

\begin{tabular}{|l|l|l|l|l|l|l|l|l|l|l|}
\hline $\begin{array}{l}\text { Experiment } \\
\text { times }\end{array}$ & 5 & 10 & 15 & 20 & 25 & 30 & 35 & 40 & 45 & 50 \\
\hline MCL & $1.12 \mathrm{~m}$ & $1.12 \mathrm{~m}$ & $1.02 \mathrm{~m}$ & $1.20 \mathrm{~m}$ & $0.98 \mathrm{~m}$ & $0.96 \mathrm{~m}$ & $0.98 \mathrm{~m}$ & $1.01 \mathrm{~m}$ & $0.90 \mathrm{~m}$ & $0.93 \mathrm{~m}$ \\
\hline HMCL & $0.79 \mathrm{~m}$ & $0.78 \mathrm{~m}$ & $0.76 \mathrm{~m}$ & $0.69 \mathrm{~m}$ & $0.68 \mathrm{~m}$ & $0.70 \mathrm{~m}$ & $0.65 \mathrm{~m}$ & $0.68 \mathrm{~m}$ & $0.64 \mathrm{~m}$ & $0.65 \mathrm{~m}$ \\
\hline
\end{tabular}

Table1: Positioning Error between MCL and HMCL

\subsection{Simulation Software Simulation}

In order to verify the wide range of wireless sensor network node location on simulation software, the simulation area is $200 \mathrm{~m} \times 200 \mathrm{~m}$, the population size (number of nodes) is 100 , the anchor node 20 , the node communication radius is $50 \mathrm{~m}$, the speed is $5 \mathrm{~m} / \mathrm{s}$, the direction of random and the initial position of the node is shown in Figure 3. The positioning of 20 beacon nodes by using the traditional MCL and HMCL, and the positioning error diagram are shown in Figure 4. The average positioning error is calculated as per Formula(5.2), un is the number of the unknown node. The number of sampling times varies with time is shown in Figure 5.

$$
e_{4}=\sum e_{2} / u n
$$

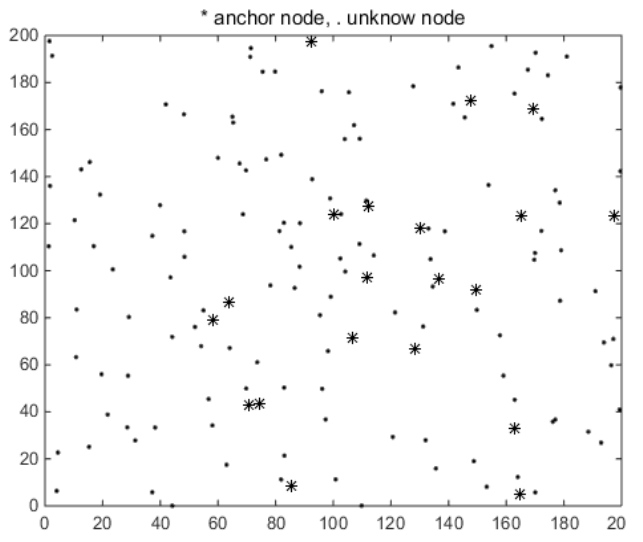

Figure 3 : Node Distribution Map

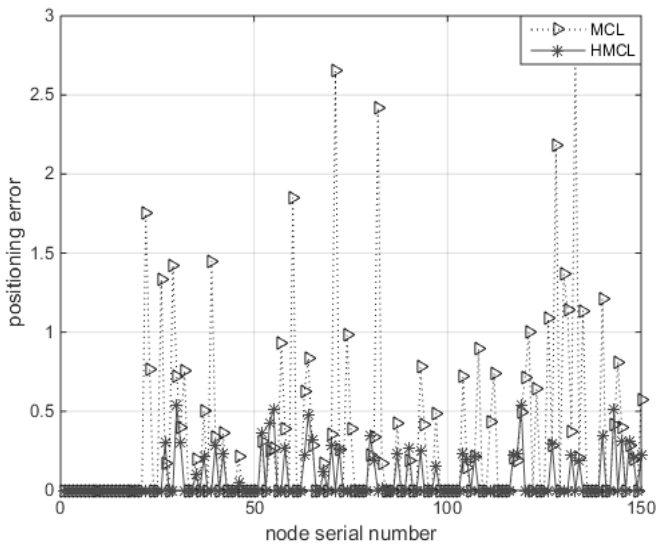

Figure 4 : Error Comparison Chart 


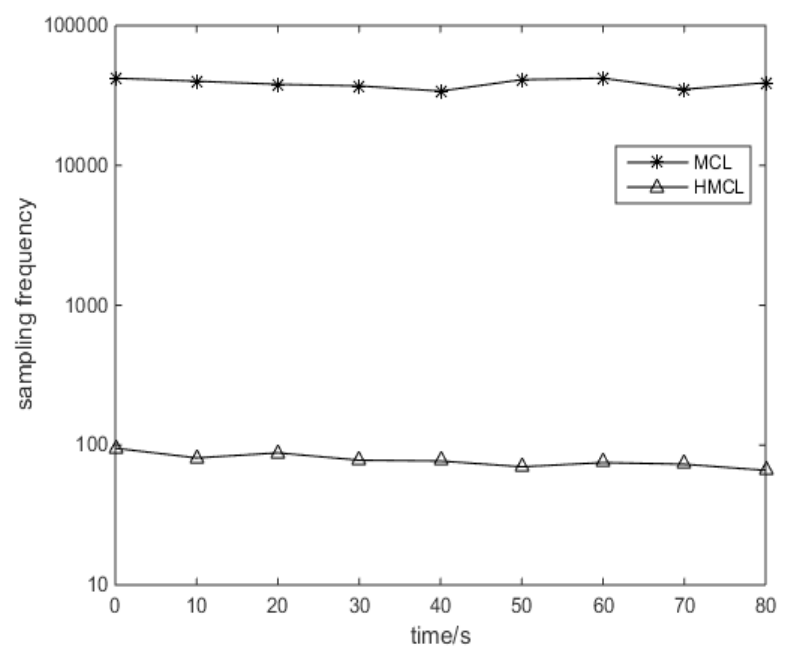

Figure 5 : Number of Sampling Times

\section{Conclusion}

The improved Monte Carlo has effectively avoided the error of RSSI data in measurement, which has greatly improved the positioning accuracy and overcome the problem of large positioning error of RSSI positioning technology. With the efficiency of predicting nodes, less sampling times and running time, the algorithm features advanced and application value to a certain extent.

\section{References}

[1] Xiaoyu Du,Lijuan Sun,Xiao Fu, et al. Localization algorithm based on minimum condition number for wireless sensor networks[J]. Journal of Electronics (China),2013,30(1):25-33

[2] Ju Mei,Di Chen,Node - Based Optimization Algorithm for Mobile Sensor Networks Based on Monte Carlo Method [J] .Journal of Sensors and Actuators, 2013,26 (5): 689-694

[3] Wei Li,YongDing.An Improved Monte Carlo Positioning Algorithm Based on RSSI[J].Computer Applications and Software(China),2013:280-284

[4] ChunYue Zhou,HongTing Zhang.An Improved Monte Carlo Location Algorithm Based on Distance Measurement $[\mathrm{J}]$.Journal of Beijing Jiaotong University,2016:63-69

[5] GuanWe Guo, DengZhong liang, GeYue tao, etc.A Location Fingerprinting Algorithm Based on RSSI Statistic Parameters Matching Estimate [J]. Key Engineering Materials, 2011:480-481

[6] L Hu ,D Evans . Localization for mobile sensor networks. In: Proc. the 10th Annual International Conference on Mobile Computing and Networking.Philadelphia: ACM Press, 2004, 45-47

[7] Rappaport T.Wireless principles and pracTice, 2nd ed. UpperSaddle River,NJ,USA: Prentice Hall PTR, 2001

[8] Xiaoyong Yan, Huanyan Qian, Zhipeng Jiang . A high accuracy localization based on RSSI measurements [J]. 2011 International Conference On Consumer Electronics,Communication and Networks (CECNet), 2011:1926-1929

[9] Doucet A,Godsill S,Andrieu C.On sequential Monte Carlo sampling methods for Bayesian filtering [J].Statistics and Computing,2000,10 ( 3) : 197-208 
[10] Mozart,Camp T,Beleng J. A survey of mobility models for RSSI network research [J]. Wireless Communications \& Mobile Computing, 2002, 2( 5) : 483-502 